\title{
La sistematización de la intervención como metodología de investigación en Trabajo Social. Importancia práctica y teórica de la fase de recogida de datos en la intervención social según experiencia del Programa de Apoyo a las Familias en Zaragoza, España
}

\author{
Elisa Esteban-Carbonell \\ Doctora en Sociología de las Políticas Públicas y Sociales. Trabajadora social \\ Universidad de Zaragoza. Zaragoza, España \\ https://orcid.org/0000-0002-1639-875X・estecar@unizar.es

\section{Nuria Del Olmo-Vicén} \\ Ph.D. Political and Social Sciences. Licenciada en Filosofía y Letras \\ Universidad de Zaragoza. Zaragoza, España \\ https://orcid.org/0000-0003-3249-1732•nolmov@unizar.es
}

\section{Resumen}

El artículo busca esclarecer, sobre la base de un estudio de caso, las limitaciones con las que se encuentra el científico social ante investigaciones en Trabajo Social. Entre los objetivos de las políticas de servicios sociales se encuentra el análisis de la realidad social para detectar situaciones de necesidad. La sistematización se erige como una metodología de investigación propia de la profesión dada la posición privilegiada de los profesionales del Trabajo Social como termómetro de la realidad social.

El objetivo del presente artículo es demostrar la utilidad de la sistematización de la práctica como método de investigación en Trabajo social; en particular, la sistematización se centra en una parte concreta de la práctica: la recogida de datos de las personas usuarias, su codificación y registro por parte de las unidades de trabajo social.

Como conclusión se ha observado que la sistematización del proceso de intervención en su fase inicial de recogida de datos de los usuarios, permite no sólo discernir qué tipo de datos son útiles y adecuados para obtener resultados positivos en la intervención, o que tipo de formación deben recibir las y los trabajadores sociales sobre el proceso de recogida; sino también permiten obtener datos primarios, para el análisis de la realidad social, confirmando la utilidad en sí del método.

Palabras clave: Sistematización; Trabajo Social; Servicios Sociales; Investigación social; Metodología.

Recibido: 27/04/2020 | Aprobado: 03/09/2020 | Publicado: 01/01/2021

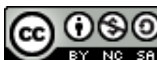

Esta obra está bajo una Licencia Creative Commons Atribución-NoComercial-CompartirIgual 4.0 Internacional.

Financiación o proveniencia del artículo: artículo derivado de la tesis doctoral "Servicios Sociales y familia: el Programa de Apoyo a las Familias en la ciudad de Zaragoza" defendida en diciembre de 2019 en la Universidad de Zaragoza, Zaragoza (España). Parte de este trabajo se ha realizado con la colaboración del Departamento de Innovación, Investigación y Universidad del Gobierno de Aragón (España).

\section{¿Cómo citar este artículo? / How to quote this article?}

Esteban-Carbonell, E., y Del Olmo-Vicén, N. (2021). La sistematización de la intervención como metodología de investigación en Trabajo Social. Importancia práctica y teórica de la fase de recogida de datos en la intervención social según experiencia del Programa de Apoyo a las Familias en Zaragoza, España. Prospectiva. Revista de Trabajo Social e intervención social, (31), 281-298. doi: 10.25100/prts.v0i31.8857. 
Esteban-Carbonell y Del Olmo-Vicén

\title{
The systematization of Intervention as a Method of Investigation in Social Work. The Practical and Theoretical Importance of the Data Collection Phase in social intervention according to experience of the Family Support Program in Zaragoza, Spain
}

\begin{abstract}
The main purpose of this work is to clarify, based on a case study, the limitations that the social scientist finds in Social Work research. Among the objectives of social services policies is the analysis of social to detect situations of need. The systematization stands as a research methodology of the profession, given the privileged position of Social Work professionals as a thermometer of social reality.

The objective of this article is to demonstrate the usefulness of the systematization of the practice as a method of research in social work; In particular, systematization focuses on a specific part of the practice: the collection of data from users, their codification and registration by the professionals of the social services centers.

In conclusion, it has been observed that the systematization of the intervention process in its initial phase of user data collection - allows not only to discern what type of data is useful and adequate to obtain positive results in the intervention, or what type of training should be given to social workers about the collection process; but also allow obtaining primary data, for the analysis of social reality, confirming the usefulness of the method itself.

Keywords: Systematization; Social Work; Social Services; Social Research; Methodology.

Sumario: 1. Introducción, 2. Referentes teóricos, 2.1 La investigación en Trabajo Social, 2.2 La sistematización como metodología de investigación en Trabajo Social, 3. Metodología, 4. Hallazgos, 5. Conclusiones, 6. Referencias bibliográficas.
\end{abstract}


Esteban-Carbonell y Del Olmo-Vicén

\section{Introducción}

El desarrollo de la investigación en Trabajo Social como actividad académica es tardío respecto a otras ciencias sociales. La carencia de continuidad en niveles formativos superiores ha condicionado a abordar la investigación en Trabajo Social desde otras disciplinas, tales como la Sociología o la Psicología Social.

No obstante, la historia del Trabajo Social va unida inevitablemente a la figura de Mary E. Richmond y es con ella con quien surge la tarea investigadora de los trabajadores sociales de forma profesional. La preocupación por sistematizar la ayuda impulsó esas primeras investigaciones, y es que "sin investigación social no es posible realizar trabajo social" (Acero, 1988, p.35) como Richmond señala en su célebre obra "Social Diagnosis". Por tanto, la investigación ha estado presente desde los orígenes del Trabajo Social, como ponen de manifiesto entre otros, Raya y Caparrós (2014). Sin embargo, no ha contado con el reconocimiento suficiente a lo largo de la historia.

Ese interés por la investigación se ha visto poco a poco relegado a un segundo plano. Se ha profundizado mucho sobre la mejora en la intervención social y se ha formado a nuestros estudiantes en ello, pero, se ha abandonado la vertiente investigadora de nuestra profesión. Kisnerman denuncia, en parte, esa "deficiente formación en investigación" (Acero, 1988, p. 36) en Latinoamérica si bien tras el movimiento de la "reconceptualización" se implantaron en varios países algunos estudios superiores especializados en investigación.

En España la situación es similar. Evidentemente son varios los factores que influyen en el desarrollo de investigaciones como pone de manifiesto, entre otros, William J. Reid. Así, la infraestructura (experiencia de los profesionales, recursos financieros, soportes institucionales y canales de divulgación), el contenido y los resultados son tres aspectos que intervienen en la producción de conocimiento (Reid como se citó en Acero, 1988, p. 37).

No sin pocas dificultades, el Trabajo Social poco a poco vuelve a adentrarse en el mundo de la investigación gracias en parte a los cambios producidos en los planes de estudio, siendo el más destacado la implantación del Espacio Europeo de Educación Superior. La titulación de Trabajo Social cambia, nuevamente, de rango y ahora pasa a tener la consideración de Grado con una duración de cuatro años de estudios, al igual que la mayoría de las otras disciplinas. Como señala Pascual (2013) nos coloca al mismo nivel que el resto de profesionales con los que compartimos los espacios de intervención social, proporcionándonos el reconocimiento que la profesión demandaba desde las primeras reivindicaciones de la Licenciatura en Trabajo Social" (p.90). Este hecho favorece la realización de estudios de postgrado, así como de doctorado y con ellos el desarrollo de las investigaciones en Trabajo Social. 
Esteban-Carbonell y Del Olmo-Vicén

El objetivo de este artículo es mostrar la importancia de la investigación en Trabajo Social a través del proceso metodológico de "sistematización de la práctica", en concreto, la sistematización de la práctica focalizada en la recogida de datos durante las fases iniciales de la intervención con familias.

El artículo se organiza en seis secciones. Tras esta introducción, el siguiente apartado trata sobre la investigación en Trabajo Social incidiendo en la importancia que tiene para la profesión. A continuación, se explica el proceso de sistematización de la práctica como método de investigación en Trabajo Social el cual contribuye tanto al desarrollo teórico de la disciplina, como a la mejora de la praxis. Posteriormente, se expone el caso concreto de análisis y la metodología llevada a cabo durante el mismo. Por último, se presentan los resultados obtenidos sintetizando las conclusiones más destacadas.

\section{Referentes teóricos}

Antes de exponer el caso de análisis, es oportuno hacer algunas observaciones teóricas; primero, una breve revisión sobre la función operativa (diagnóstica) y básica (evaluativa) de la investigación en la disciplina; y segundo, una reflexión sobre los objetivos y fundamentos de la sistematización de la práctica como método de investigación.

\subsection{La investigación en Trabajo Social}

Teniendo en cuenta que "la función de la investigación en Trabajo Social es contribuir al desarrollo de un cuerpo de conocimientos comprobados que sirvan a los fines y medios del Trabajo Social en todas sus ramificaciones" (Marco y Tomás, 2013, p. 236). ¿qué significa investigar en Trabajo Social?

Rubiól (1973, p. 87) distinguía dos direcciones de la investigación en Trabajo Social.

a. Investigación para Trabajo Social: para conocer el campo en el que se actúa y el objeto concreto que se estudia. También se llama investigación operativa (ejemplo: estudio sobre necesidades de una comunidad, sobre problemas de individuos, sobre características de los individuos con los que se trabaja, etc.).

b. Investigación sobre Trabajo Social: va dirigida hacia el propio trabajo profesional. También se llama investigación básica y tiene que salir de la evaluación constante y sistemática del trabajo que realiza el Trabajo Social (ejemplo: estudio para medir los servicios ofrecidos, su adecuación y eficacia, sobre la metodología, etc.).

La National Association of Social Workers, a mediados de los años 50, así como numerosos autores, entre otros William J. Reid (citado en Acero, 1988) enuncian y clasifican 
Esteban-Carbonell y Del Olmo-Vicén

los diferentes tipos de investigación en el campo del Trabajo Social. En todas sus vertientes, la investigación se realiza para orientar la práctica profesional, bien para detectar nuevos campos de acción a partir de diagnósticos de necesidades, por ejemplo, o bien para mejorar la práctica profesional. En cualquier caso, es indiscutible la relación existente entre investigación e intervención.

Se puede afirmar, por tanto, que la investigación es una pieza clave es nuestra profesión ya que guía la intervención dotándola de contenido teórico y validez científica y permite discernir entre "investigación fundada" e "investigación indiscriminada" (Ramos, 2013, p. 68). Como señalan Raya y Caparrós (2014), “son varias las voces que desde el Trabajo Social apuntan a la importancia de la investigación" (p. 175). Por tanto, investigación e intervención constituyen "dos estadios dentro del proceso metodológico".

En ese vínculo existente entre la práctica y la investigación, Cifuentes (2013) argumenta su tesis bajo la idea de que la práctica profesional ha de cumplir con dos condiciones básicas. Por un lado, "ha de ser una práctica fundamentada en el conocimiento crítico de lo social, (es decir, con fines transformadores, producidos por la profesión y por las ciencias sociales) y en los resultados de la investigación social" (p. 168) y, por otro lado, "debe ser (...) coherente con el compromiso ético del Trabajo Social en relación con la justicia social" (Cifuentes, 2013, p. 167).

Por otra parte, y siempre desde la idea de la necesidad de investigar para intervenir, Barbero (2006) señala que las investigaciones de Trabajo Social "conectan con la perspectiva operativa" (p.48-49), es decir, la generación de conocimiento está dirigida a la actuación o bien a la redefinición de la intervención. Ambos objetivos planteados por Barbero (teoría para hacer práctica y teoría para redefinir la práctica) están relacionados con los tipos de investigación planteados anteriormente por Rubiól (1973).

Si nos detenemos en la investigación sobre el Trabajo Social o, siguiendo la idea de Barbero en "investigación para la redefinición de la intervención", vemos cómo la investigación trata, de alguna manera, de evaluar las prácticas profesionales tratando de cribar entre aquellas que se ajustan a las necesidades y los problemas de aquellas que no resultan del todo útiles, es decir, de perseguir la eficacia en nuestras intervenciones. Por tanto, es necesario "un proceso continuo de retroalimentación" como pone de manifiesto (Vázquez, 2013, p. 54).

Esto implica, para empezar, la continua actualización de los nuevos avances por parte de los profesionales. Los trabajadores sociales deberían guiar sus actuaciones apoyándose en todo lo que se escriba en relación a la mejora de la intervención. Pero también, es necesario dotar de contenido científico a la práctica, es decir, recopilarla y evaluarla. 
Esteban-Carbonell y Del Olmo-Vicén

Ahora bien, ¿cómo es posible llevar a cabo este tipo de investigación?

Entre las posibilidades de conocimiento de la práctica profesional, la sistematización, entendida como un tipo de investigación cualitativa, viene considerándose hace ya varias décadas como una modalidad prometedora para reconstruir críticamente los saberes que se producen y que pueden ser aprendidos de la práctica (...). (Cifuentes, 2013, p. 177)

\subsection{La sistematización como metodología de investigación en Trabajo Social}

La investigación en Trabajo Social se nutre del corpus metodológico desarrollado por disciplinas afines, especialmente desde la metodología de la Sociología, de la Psicología social y de la Antropología. En particular la metodología de estas disciplinas ha sido útil desde el planteamiento del Trabajo Social como nivel micro de las políticas sociales; es decir, dado que uno de los objetivos de las políticas de servicios sociales es el análisis de la realidad social para detectar situaciones de necesidad, estas técnicas han permitido diagnósticos eficaces para la intervención. En este sentido, tradicionalmente se ha partido del planteamiento del objetivo a investigar, y la posterior elección de las técnicas de recogida y análisis de datos.

Sin embargo, la posición privilegiada del trabajador social como termómetro de la realidad social, empuja a centrarnos en el tipo de datos que necesitamos por encima de los objetivos a investigar. En este punto la sistematización de la práctica en los centros de servicios sociales constituiría el punto de partida de la investigación social, cuyo fin último nos conduce a un diagnóstico preciso en el diseño de políticas sociales a nivel micro.

En cierto modo es una propuesta de retorno a los orígenes de la profesión, cuyo análisis de los estudios de caso tanto contribuyó al desarrollo de la profesión y de otras ciencias sociales. Reconociendo la aportación diaria y casi invisible que los trabajadores sociales hacen con el análisis de la realidad social.

En este sentido, como pone de manifiesto Bueno (2013), el Trabajo Social tiene que apostar por desarrollar prácticas reflexivas que sistematicen y teoricen las experiencias vividas en las prácticas profesionales, ya que los datos obtenidos permiten crear alternativas de intervención al mismo tiempo que construir desarrollos teóricos para la propia profesión. A pesar de ello, existen ciertos problemas en torno a la metodología de la sistematización siendo uno de ellos la indefinición de sus fronteras entre ésta, la evaluación y la investigación social.

La sistematización se centra en la dinámica de los procesos, la evaluación en los resultados. En la evaluación no se reflexiona sobre el proceso vivido, sino que se miden los 
Esteban-Carbonell y Del Olmo-Vicén

resultados en relación con los objetivos planteados. Un punto en común de ambas es que debe existir una retroalimentación y el fin es mejorar los resultados de la intervención. Además, la investigación social teoriza sobre aspectos de la realidad enriqueciendo la práctica con nuevos elementos teóricos, por lo tanto, la reflexión no puede separarse de la investigación en sí misma; por esto, la reflexión es parte intrínseca tanto de la investigación como de la sistematización. Sin entrar en detalle sobre las diferentes propuestas de sistematización, parecen evidentes algunas cuestiones básicas y comunes. Destacamos así cuatro aspectos fundamentales, la sistematización implica:

- Reconstrucción ordenada de la práctica: ordenar y organizar el proceso y los resultados, así como el sentido o el significado que el proceso ha tenido para los actores participantes en ella Martinic (como se citó en Sandoval, 2001, pp. 115116).

- Producción de conocimientos: la sistematización es una articulación entre teoría y práctica y sirve tanto para mejorar la práctica como para enriquecer y modificar el conocimiento existente.

- Conceptualización de la práctica: uno de los propósitos de la sistematización para poner en orden todos los elementos que ahí intervienen, articulados en un todo en el que cada una de las partes sea consciente de su razón de ser, sus potencialidades y sus limitaciones y una búsqueda de coherencia entre lo que se quiere hacer y lo que se puede hacer.

- Participación: proceso de creación participativa de conocimientos teóricoprácticos.

Es decir, "la sistematización es una interpretación crítica de la práctica que, a partir de su ordenamiento y reconstrucción, descubre o explicita la lógica del proceso vivido, los factores que han intervenido en dicho proceso, cómo se han relacionado entre sí, y por qué lo han hecho de este modo" (Sandoval, 2001, pp. 116-117).

Pero la contribución de la sistematización de la práctica en Trabajo Social va mucho más allá de su contribución a la mejora de la praxis, la sistematización se erige como punto de partida fundamental en la investigación social en general, y para y sobre el Trabajo Social en particular por varias razones:

En primer lugar, la sistematización permite perfilar diferentes objetos de estudio, es decir, nos permite establecer el objeto de estudio en torno a individuos o grupos de población concretos (bien, usuarios de servicios bien, población con necesidades especiales); también puede focalizarlo en las relaciones de los profesionales del Trabajo Social y/o de 
Esteban-Carbonell y Del Olmo-Vicén

otras profesiones que desarrollan su actividad junto con éstos. De igual modo a partir de la sistematización de la práctica se puede focalizar el objeto de estudio en las interrelaciones que se generan entre los distintos profesionales y los usuarios o beneficiarios de los programas. Incluso pueden erigirse en objeto de la investigación las propias instituciones u organizaciones que promueven actividades de acción social, y como no, el contexto o contextos en los que se desarrolla dicha práctica (social, cultural, institucional, administrativo-legal y político).

Por lo tanto, la sistematización abre un campo de posibilidades de investigación que permitirá una reflexión desde distintas perspectivas de la realidad social, enriqueciendo por sí misma, no solo a la práctica y a la teoría del Trabajo Social, sino también a otras disciplinas. Merece especial atención este aspecto dado que dota a la investigación de la apertura necesaria para contribuir al conocimiento teórico en diferentes niveles (perfiles de usuarios, praxis profesional, nivel institucional, contexto, etc.).

En segundo lugar, la sistematización permite aunar estrategias metodológicas, cuantitativas y cualitativas, en las técnicas y en los métodos, para producir y tratar la información. Así a través de la sistematización de la práctica, realizada durante un periodo suficiente y adecuado de años, se puede generar un conjunto de datos primarios y secundarios, utilizables tanto para la disciplina del Trabajo Social en cuanto la intervención, como desde el ámbito de la Sociología para el análisis de fenómenos sociales que de otra manera no hubieran aflorado.

En tercer lugar, en definitiva a través de la sistematización de la práctica se contribuye a la generación de bases de datos que pueden ofrecer tanto una radiografía social del momento elegido, así como permitir de manera continua y sistemática el desarrollo de estudios longitudinales, de gran utilidad no solo para la implementación de políticas sociales a nivel meso y micro (programas y carteras de servicios sociales), sino también para trazar los planes nacionales de atención a diversos grupos sociales y particularmente a contribuir desde la ciencia de la administración al desarrollo de un sistema de servicios sociales y, en general, de sistemas de bienestar, más eficientes y sostenibles. Además de su contribución a la calidad de los mismos, dada la relación implícita que se establece entre la sistematización de la práctica y la evaluación de los programas desde varias perspectivas ("accountability", etc..).

Esta utilidad de la sistematización de la práctica para otras disciplinas afines, también contribuirá a elevar el posicionamiento de la disciplina entre las ciencias sociales, a empoderar el Trabajo Social y a sus profesionales, como bien expresan algunos autores: “El Trabajo Social tan poco valorizado muchas veces por el resto de las disciplinas sociales, le puede aportar a las mismas el nivel de acción experimental que cotidianamente realiza con los sujetos sociales y desde el cual podría aportar elementos, desde los cuales, las ciencias 
Esteban-Carbonell y Del Olmo-Vicén

sociales dejarían de ser meras descripciones o especulaciones sobre lo que ocurre en el mundo" (Di Carlo y Elda, 2005, p. 34).

\section{Metodología}

Como se ha mencionado previamente, el objetivo del presente artículo es demostrar la utilidad de la sistematización de la práctica como método de investigación en Trabajo Social. En particular, la sistematización se centra en una parte concreta de la práctica: la recogida de datos de los usuarios, su codificación y registro por parte de las unidades de Trabajo Social.

Como hipótesis de partida, se plantea que la sistematización del proceso de intervención en su fase inicial de recogida de datos, permite no sólo discernir qué tipo de datos son útiles y adecuados para obtener resultados positivos en la intervención, o qué tipo de formación deben recibir los trabajadores sociales sobre el proceso de recogida; sino que también permiten obtener datos primarios para el análisis de la realidad social, confirmando la utilidad en sí del método.

\section{Caso de estudio: la generación de datos a través de la sistematización de la práctica en el Programa de Apoyo a las Familias}

De acuerdo con lo anteriormente mencionado, los datos se extraen del proceso de sistematización de la intervención con familias dentro del Programa de Apoyo a las Familias (PAF) correspondiente al periodo 2009-2017; en particular aquellos pertenecientes a la fase inicial de la intervención, fase en la que se registran los datos de los usuarios participantes en el programa.

El Programa de Apoyo a las Familias se puede catalogar como un programa psicoeducativo dirigido a familias que busca mejorar las competencias parentales de cuidado, educativas y de comunicación hacia sus hijos e hijas menores de edad, cuando por diversos factores no pueden desempeñarlas por sus propios medios. Se trata de un servicio implementado desde principios de los años 90 en la ciudad de Zaragoza. Concretamente, el entonces denominado Programa de Apoyo psico-socio-educativo a la Infancia y la Familia se puso en marcha en 1991 en los barrios de Las Fuentes, San José y Torrero y posteriormente, implantándose en el resto de barrios zaragozanos.

Inicialmente se seleccionó una muestra aleatoria de 24 intervenciones del PAF, desarrolladas en tres centros municipales de servicios sociales de la ciudad de Zaragoza, concretamente CMSS Delicias, CMSS La Magdalena y CMSS San José. La elección de la intervención en estos centros busca obtener distintos perfiles: primero, un centro donde han 
Esteban-Carbonell y Del Olmo-Vicén

existido los PAF breves (CMSS Delicias), es decir, intervenciones familiares más cortas de duración a lo estipulado en el programa enmarcadas dentro del propio PAF. Segundo, un centro con una importante trayectoria en intervenciones familiares en grupo o PAF grupal (CMSS La Magdalena). Y, por último, un centro en el que el PAF ha sido implementado tal y como recoge el documento del programa (CMSS San José).

En esta fase de recogida de información de los expedientes, se tomaron datos disponibles en las respectivas bases de datos SSCC, así como en el expediente en papel. En particular, respecto a la base de datos SSCC, se registró toda la información disponible en el epígrafe denominado "datos de cabecera" que reúne datos generales (fecha de alta, fecha histórico, familia, entre otros), nombre del usuario principal, dirección del domicilio, expediente (número, centro, etc.), ingresos comunes a la parrilla (usuario titular, prestación, etc.), e ingresos familiares (importe rentas mobiliarias anuales, importe rentas inmobiliarias anuales e importe otros ingresos anuales).

Al mismo tiempo se registró información relativa al apartado "datos vivienda" (tipo vivienda, pérdida, régimen de tenencia, coste mensual -alquiler/hipoteca-, barreras arquitectónicas accesos, falta iluminación natural, deteriorada, falta ventilación, observación equipamiento, habitaciones, $\mathrm{m}^{2}$ / persona, superficie $\mathrm{m}^{2}$, fecha compra/alquiler, coste comunidad, aceptable, malas condiciones, amenaza de ruina, inadecuada distribución del espacio y barreras arquitectónicas vivienda), así como datos sobre el equipamiento (agua caliente, ascensor, agua corriente, calefacción casa entera, ducha, electricidad, frigorífico, gas, lavadora automática, teléfono, W.C. y observaciones).

Además de los datos anteriores de carácter general, la base de datos recoge cada una de las intervenciones que se llevan a cabo dentro de cada expediente. En este sentido, respecto al PAF, se recopiló información de los siguientes apartados:

- Detalle datos intervención: número de intervención, UTS1, fecha inicio, fecha fin, implica gestión administrativa, profesional, estado, sector, recurso, valoración.

- PAF: código prestación, estado.

- Solicitud: fecha solicitud, apellidos y nombre, documento, problemática.

- Valoración PAF: aspectos laborales (situación laboral familiar, responsabilidad familiar, actitud búsqueda empleo, realización gestiones), aspectos económicos (situación económica, nivel ingresos familia, renta per cápita, manejo dinero), y

1 Unidad de Trabajo Social. 
Esteban-Carbonell y Del Olmo-Vicén

aspectos educativos (adecuación necesidades educativas, condiciones estudio, asistencia colegio, rendimiento escolar).

- Alta: primera propuesta, alternativa, hipótesis, fecha alta, fecha presentación familia, fecha finalización programa.

- Datos de baja: fecha valoración familia, fecha informe final, motivo baja, fecha baja.

- PAF: objetivos (y su valoración), riesgos (y su valoración), horarios, profesionales

$\mathrm{Y}$, respecto a los expedientes en papel es importante señalar las acciones de recogida de datos que se desarrollan respecto a los documentos siguientes:

- Solicitud prestación servicio, se recogieron los siguientes datos: $\mathrm{n}^{\circ}$ SIUSS2, UTS, fecha, composición familiar ${ }^{3}$, origen ${ }^{4}$, descripción de la problemática, indicadores de riesgo, indicadores priorizados/primeras propuestas de intervención, aspectos positivos que favorezcan la intervención, actuaciones realizadas.

- Programación inicial/general, se recogieron los siguientes datos: $\mathrm{n}^{\circ}$ SIUSS, CMSS, fecha de la programación, miembros del equipo, objetivos generales, objetivos específicos por áreas ${ }^{5}$ (laboral/económica; escolar; vivienda; educación para la salud; legal, jurídica y administrativa; organización del hogar y economía doméstica; relaciones familiares; relaciones sociales), temporalización, aspectos a observar, primeras hipótesis de trabajo.

- Evaluación por áreas, se recogieron los siguientes datos: nº SIUSS, CMSS, fecha, área 1 laboral, económica y de inserción; área 2 educativa (escolar); área 3 vivienda; área 4 educación para la salud; área 5 legal, jurídica y administrativa; área 6 organización del hogar y economía doméstica; área 7 relaciones familiares; área 8 relaciones sociales.

2 Sistema de Información de Usuarios/as de Servicios Sociales desarrollado desde 1994 por el actual Ministerio de Sanidad, Consumo y Bienestar Social en colaboración con las Comunidades Autónomas (Convenios de Colaboración).

3 El nombre de esta variable es de elaboración propia con el fin de facilitar la recogida de información. En la solicitud de la prestación figura un cuadro donde se escriben los nombres y los apellidos junto a sus fechas de nacimiento y el grado de parentesco respecto al usuario principal de todos los miembros que componen la unidad familiar.

4 El nombre de esta variable es de elaboración propia al observar cómo en algunos expedientes se incluía la variable origen en algún momento de la ficha.

$5 \mathrm{El}$ orden de las áreas no es el mismo en todos los expedientes posiblemente a cambios en los documentos en función de los años de intervención del PAF. 
- Programaciones mensuales, se recogieron los siguientes datos: $\mathrm{n}^{\circ}$ SIUSS, UTS, mes, período, educador, objetivos específicos, actividades, desarrollo.

- Informe final, se recogieron los siguientes datos: $n^{\circ}$ SIUSS, CMSS, miembros del equipo, fecha, fecha de alta, fecha de salida, motivo de la salida, resumen objetivos por áreas, actividades tipo, evolución de los objetivos ${ }^{6}$, valoración. En algunos casos también se incluye un apartado sobre el proceso de intervención y sobre la evaluación por áreas.

- Informe de estudio-valoración, se recogieron los siguientes datos: datos de identificación; datos de identificación familia; otros datos; composición familiar; sobre la demanda; situación laboral y económica; situación de la vivienda; organización y equipamiento; aspectos sanitarios y de hábitos de higiene; situación escolar; relaciones familiares; relaciones sociales; aspectos legales y judiciales.

Posteriormente se recogió la información obtenida por los 13 trabajadores sociales que habían realizado la sistematización de la intervención con las familias en esta etapa inicial de recogida de datos.

\section{Hallazgos}

Hemos comprobado cómo a partir de la sistematización -de las acciones y documentos de trabajo- realizada en la fase inicial de la intervención dentro del programa PAF, se ha podido primero, extraer valiosa información sobre los datos que se recogen de las personas usuarias comprobando que el proceso de recogida de datos en la intervención es mejorable, dado que se evidencian tanto los déficits en los datos recogidos a las personas usuarias del programa, como los fallos respecto al proceso en sí de recogida de datos.

Por una parte, se han detectado vacíos de información en la base de datos (SSCC) utilizada en los centros municipales de servicios sociales. El SSCC comprende -de cada uno de los expedientes- información general sobre la unidad familiar y un listado de todas las intervenciones que se han llevado a cabo con dicha familia. Se puede ir entrando en cada una de las mismas y obtener información concreta sobre el programa, servicio o prestación de la que la familia ha sido usuaria o beneficiaria.

6 La evolución de los objetivos puede encontrarse por áreas específicas siguiendo las áreas contempladas en otro tipo de documentos o bien a partir de las siguientes variables: indicadores iniciales, indicadores finales y resistencias al cambio. No todos los epígrafes están en todos los documentos. 
Sin embargo, apenas dos expedientes, del total de 24 que componen la muestra aleatoria, ofrecen algo de información sobre estos apartados generales. Este hecho conduce obligatoriamente a la siguiente cuestión: ¿se desestima recoger tanta información en los expedientes porque no es necesaria para la intervención y su seguimiento? Los y las profesionales que intervienen en la práctica, ponen de manifiesto que la información recogida no procede dado que la población usuaria de los servicios sociales es un tipo de población que cambia con facilidad de situación económica, laboral, habitacional, etc. lo que obligaría a una actualización constante de este tipo de datos introducidos en la base de datos.

Junto a estos datos de carácter general, la base de datos también ofrece la posibilidad de introducir información relativa a cada una de las intervenciones, entre las que se encuentra el PAF. De igual modo, la fase de explotación de la muestra aleatoria de los procesos de intervención, ha permitido dar cuenta de los fallos en la recogida de información dentro del programa; cada profesional recoge críticas sobre aspectos de la base de datos como la falta de concordancia con el documento marco y su excesiva rigidez. En este sentido, la información de obligada cumplimentación (como, por ejemplo, los objetivos) no se corresponde con la recogida en el documento marco, al mismo tiempo que la base de datos no permite al profesional perfilar la información. Es decir, por ejemplo, respecto a los objetivos, hay un listado cerrado que impide que el o la profesional redacte objetivos concretos u operativos.

Por otro lado, hay apartados optativos o, más bien, que no son de obligada cumplimentación lo que da lugar a que todos los expedientes ofrezcan información adicional. Cada centro e incluso cada profesional ha adoptado dinámicas distintas al respecto, de modo que se encuentran centros -e incluso expedientes- con únicamente la información obligatoria junto a otros expedientes con mayor número de datos. Un ejemplo se encuentra en la cumplimentación de las fechas. De las fechas que contempla la base de datos (fecha de solicitud, fecha de alta, fecha de presentación familia, fecha finalización programa, fecha valoración familia, fecha informe final, fecha baja), no todas están cumplimentadas ni todas lo están bajo el mismo criterio. El análisis de la muestra pone en evidencia que algunas fechas están sin cumplimentar junto a otras que coinciden evidenciando su no correspondencia con la realidad del proceso. Las educadoras y educadores familiares revelan, en este sentido, las diferencias respecto a los criterios en su cumplimentación.

Asimismo, hay un apartado que recoge la información sobre la vivienda denominada "datos vivienda" y que comprende además de datos de identificación y datos específicos sobre la vivienda, datos sobre su equipamiento. Señalar que sólo un expediente del total de 24 ofrece información sobre los datos de la vivienda y sobre el equipamiento de la misma, anotándose, en este sentido, algunas observaciones al respecto. Específicamente el tipo de 
Esteban-Carbonell y Del Olmo-Vicén

vivienda (en régimen de alquiler), la cuota mensual y el número de habitaciones de la vivienda.

Igualmente, el SSCC contiene datos sobre la parrilla familiar, es decir, nombre y apellidos de cada uno de los miembros de la unidad familiar, así como su fecha de nacimiento y el parentesco que guardan con la persona usuaria principal. Se trata de datos de cumplimentación obligatoria y, por tanto, todos los expedientes analizados los recogen.

Otro apartado del SSCC es el seguimiento de las intervenciones. Supone un apartado central dado que se recoge -en un espacio pequeño- cómo ha transcurrido la intervención con la familia. Este apartado es cumplimentado por cualquier profesional o técnico que trabaje con esta unidad familiar, bien sea de manera directa como indirecta. Además de la descripción, aparece la fecha y el nombre propio del profesional que lo ha cumplimentado. Se trata de un espacio muy utilizado por las y los profesionales ya que es el único que permite recoger el proceso de intervención. No obstante, hay un límite de palabras por cada "entrada" de seguimiento, por lo que es habitual encontrarse varias "entradas" para referirse a una sola cita. Por otra parte, supone un espacio visible para todos los profesionales que trabajan con la familia y, por tanto, pueden hacer uso del mismo, generando, en este sentido, dificultades para poder seguir el "hilo" del proceso de intervención. Algo similar sucede con los documentos en papel; la base de datos no genera la documentación de forma automática, por lo que el acceso digital a los documentos queda supeditado al hecho que la y el profesional haya decidido adjuntar dicha documentación al expediente.

Por otra parte, siguiendo con los déficits en la recogida ordenada de la información y en relación con la idea anterior, hay diferencias notables respecto a la documentación en papel recogida en cada uno de los expedientes. El análisis de los 24 expedientes elegidos al azar dentro de nuestra sistematización reveló la existencia de los siguientes documentos en papel:

- Solicitud prestación servicio

- Programación inicial/general

- Evaluación por áreas

- Programaciones mensuales

- Informe final

- Informe de estudio-valoración 
Sin embargo, de los 24 expedientes analizados, 6 expedientes no contaban con el documento de solicitud y aquellos que sí tenían el documento habían cumplimentado todos los apartados a excepción de uno que tenía un apartado sin rellenar.

De los 24 expedientes analizados, sólo 10 de ellos cuentan con algún documento de programación inicial/general en cualquier formato7. Por otra parte, sólo 6 casos de los 24 analizados cuentan con el documento evaluación por áreas. Cabe señalar que entre los expedientes que sí que cuentan con la documentación, tres de ellos han cumplimentado la evaluación en dos momentos temporales, y en otro caso encontramos tres evaluaciones.

Respecto a las programaciones mensuales, 13 casos del total de 24 analizados cuentan con los documentos, y en varios de ellos con varias programaciones. Del total de 24 expedientes analizados, 9 de ellos cuenta con el documento de informe final. Sólo en 3 expedientes del total de 24 analizados se ha encontrado el informe de estudio-valoración. De todos estos documentos, la ficha de solicitud de la prestación y el informe final han sido los más numerosos, encontrándose de forma extraordinaria el resto de documentos.

Los resultados obtenidos en esta fase de recogida de información revelan una falta de homogeneización respecto al uso de la documentación propia del PAF. Cada centro (e incluso cada educador o educadora en algunos centros) ha establecido unos criterios en cuanto al proceso de recogida de datos de las personas usuarias en la primera fase de la intervención.

\section{Conclusiones}

De la presente investigación se pueden exponer dos tipos de conclusiones, desde un plano empírico y desde un plano teórico. Las conclusiones empíricas se extraen sobre la información que aporta la sistematización de la fase inicial -o fase de recogida de datos-, en la intervención con familias en el programa PAF. Por su parte, las conclusiones teóricas ofrecen una reflexión sobre la sistematización como metodología de la investigación en Trabajo Social.

Antes de nada, hay que poner en evidencia la ausencia de diferencias entre los perfiles de los centros municipales seleccionados. En este sentido, la variable "centro" no condiciona la presencia de las conclusiones extraídas.

7 En algunos expedientes se denomina "programación inicial", en otros "programación general", en otros "programación inicial/general" señalando si es inicial o general. En todos los casos las variables recogidas son las mismas. 
Esteban-Carbonell y Del Olmo-Vicén

La sistematización de la práctica en su fase inicial de recogida de datos nos ha aportado información suficiente para valorar los siguientes puntos de mejora: sobre el tipo de datos a recoger, el diseño de los instrumentos de recogida y la formación de las y los profesionales sobre la fase de recogida de datos, como procesos clave dentro de la intervención.

Respecto a los datos, hay dos aspectos importantes a destacar. Por un lado, la recogida de información en la base de datos informática y, por otro lado, la recogida de información manual disponible en los expedientes sociales.

La sistematización de la práctica pone de manifiesto la falta de instrucciones claras al respecto. Más allá de la formación inicial a las y los profesionales de los centros, no hay más indicaciones que permitan utilizar de forma homogénea la base de datos, lo que da lugar a variaciones considerables en los datos consultados durante la investigación y que queda palpable, por ejemplo, a partir del "baile" de fechas en los distintos expedientes. Pero existen otros muchos ejemplos que permiten justificar la necesidad de contar con instrucciones claras y, sobre todo, comunes.

En relación con los expedientes sociales en su soporte físico, a pesar que en el documento marco se señalan los documentos de obligado cumplimiento, así como los opcionales, estas fases de la investigación han permitido identificar la disparidad de documentos utilizados por las y los profesionales o, al menos, conservados en los distintos expedientes sociales. Bien es cierto, que la fase de trabajo de campo ha coincidido con un momento de redefinición del programa y, más concretamente, de redefinición del documento marco. Habrá que esperar un tiempo para comprobar si se ha homogeneizado el uso de los distintos documentos $\mathrm{y}$, sobre todo, si se mantienen disponibles en el expediente social.

Las diferencias no solo residen en el tipo de documentos utilizados, ya que su cumplimentación asimismo varía de un expediente a otro, de profesional a profesional, así como de un centro municipal a otro. De nuevo, se requieren instrucciones claras que permitan la homogeneización de la recogida de la información.

Esta realidad respecto a los datos obligatoriamente debe conducir a una reflexión profunda sobre dos aspectos. Por un lado, es urgente pensar qué tipo de datos son necesarios recopilar (ya sea en formato digital, como en papel) y por qué. Algo que han puesto de manifiesto las y los profesionales de los centros de servicios sociales, afirmando que la recogida de cada dato debe tener un objetivo, un fin concreto de cara a los resultados de la intervención. No es otra cosa que pensar en la utilidad de los mismos y el uso posterior que se va a hacer. ¿Para qué queremos recoger datos? Ésta debe ser una pregunta clave a la que dar respuesta. 
Desde el punto de vista de la sistematización como método de investigación, en general, se puede concluir que la importancia de la sistematización de la práctica de cara a su utilidad en la investigación de Trabajo Social -con fines tanto de mejora de la práctica como de construcción de la disciplina- radica en concretar algunos aspectos básicos, aunque ampliables en función del propio objeto de estudio.

Primero, sobre el objeto de la sistematización: dado que el proceso de sistematización debe ir unido a una reflexión acerca del mismo. Es decir, la sistematización debe ser justificada, para ello será necesario preguntarnos qué queremos sistematizar y por qué. Habrá que determinar si queremos sistematizar un aspecto concreto de la intervención o toda ella y evidentemente saber la razón de ser de la sistematización, el motivo que empuja a llevar a cabo la sistematización. Se busca sistematizar para mejorar, para rendir cuentas.

Segundo, sobre los objetivos de la sistematización: debemos plantearnos la pregunta ¿por qué se quiere sistematizar? Nuestro objetivo puede ser comprender mejor lo que hacemos, pero también mejorar lo que hacemos. Es preciso clarificar los objetivos de este proceso.

Tercero, sobre el contexto de la práctica. Antes de llevar a cabo el propio proceso de sistematización hay que conocer el contexto en el que se desarrolla dicha práctica. Conocer sus entresijos y sus distintos elementos: políticos, culturales, sociales, etc. No podemos abordar la sistematización sin conocer bien la institución y los tipos de intervención que se llevan a cabo. Así como complementarlo con el conocimiento del contexto político, económico, social y cultural del mismo.

Cuarto, sobre el proceso de interpretación y reflexión. Como se ha mencionado anteriormente, lo importante no es la descripción sino la interpretación de forma, evidentemente, crítica. Se trata de conocer qué pasó y por qué pasó. Partiendo de la descripción se debe realizar un análisis y una síntesis a través de una interpretación crítica del proceso. Para ello, se deben desgranar los aspectos esenciales del proceso, es decir, penetrar en cada una de las partes que conforman la intervención tratando de detectar las tensiones y/o contradicciones que pudieron marcar el proceso. No solo esto, sino también se deben interpretar las relaciones que se establecieron, los aspectos facilitadores del proceso, los obstáculos, etc. Los aspectos llamémosles "negativos" del proceso se pueden analizar tanto desde una perspectiva individual como grupal o institucional y evidentemente ver cómo se afrontaron por todas las personas que estuvieron implicadas. Además, se debe reflexionar acerca de las necesidades, esto es, ver si las necesidades iniciales se mantuvieron, se solventaron, si surgieron otras, etc. En resumen, se trata de ver la secuencia y lógica con la que se fueron dando las variaciones en la intervención. 
Esteban-Carbonell y Del Olmo-Vicén

Resumiendo, se demuestra que la sistematización de la práctica es un método muy útil de investigación en Trabajo Social, que permite la obtención de datos orientados a la mejora de la praxis, así mismo conduce a la reflexión previa a la generación de una metodología de investigación propia del Trabajo Social fundamentada en los procesos de intervención.

\section{Referencias bibliográficas}

Acero, C. (1988). La investigación en Trabajo Social. Cuadernos de Trabajo Social, (1), 36-46. Barbero, M. (2006). La autogestión del Trabajo Social y la perspectiva del investigador. Cuadernos de Trabajo Social, (19), 43-54.

Bueno, A. M. (2013). Producción de conocimiento sobre investigación en Trabajo Social. Trabajo Social, (15), 125-141.

Cifuentes, M. R. (2013). Formación en Trabajo Social e investigación: una relación insoslayable de cara al siglo XXI. Trabajo Social, (15), 165-182.

Di Carlo, E., y Elda, B. (2005). Importancia de la investigación para el Trabajador Social profesional. Tendencias y Retos, (10), 33-37.

Marco, M., y Tomás, E. (2013). La investigación en y para la intervención social: la aplicación al Trabajo Social. En M. Miranda. (Coord.), Aportaciones al Trabajo Social (pp. 223-248). Zaragoza, España: Prensas de la Universidad de Zaragoza.

Pascual, M. T. (2013). Reflexiones sobre la evolución de la investigación en el ámbito académico. En A. J. Olalde e I. López, (Coords.), VI Jornada de Trabajo Social, Investigación y Trabajo Social: Dialogando desde la intervención (pp. 81-92). País Vasco: Universidad del País Vasco, EHU.

Ramos, C. (2013). La producción de conocimiento en Trabajo Social: un proceso interactivo. En A. J. Olalde. e I. López (Coords.), VI Jornada de Trabajo Social, Investigación y Trabajo Social: Dialogando desde la intervención. (pp. 65-78.) País Vasco: Universidad del País Vasco, EHU.

Raya, E., y Caparrós, N. (2014). Del objeto de estudio para la intervención en Trabajo Social. AZARBE, Revista Internacional de Trabajo Social y Bienestar, (3), 173-179.

Rubiól, G. (1973). Investigación en Trabajo Social. En Federación Española de Asociaciones de Asistentes Sociales, Memoria del II Congreso Nacional de Asistentes Sociales (pp. 83100). Madrid: Federación Española de Asistentes Sociales.

Sandoval, A. (2001). Propuesta Metodológica para sistematizar la práctica profesional del trabajo social. Buenos Aires, Argentina: Espacio Editorial.

Vázquez, O. (2013). Investigar para fortalecer la dimensión política del Trabajo Social. De la sistematización de la práctica a la investigación. En A. J. Olalde. e I. López (Coords.), VI Jornada de Trabajo Social, Investigación y Trabajo Social: Dialogando desde la intervención (pp. 51-64). País Vasco: Universidad del País Vasco, EHU. 


\section{OTROS ARTÍCULOS DE PROSPECTIVA No. 31 DE 2021}

\section{PRESENTACIÓN}

Presentación. Reflexiones sobre desafios al publicar sistematizaciones

Rosa María Cifuentes-Gil

\section{EDITORIAL}

Reflexiones sobre Trabajo Social: aportes de la Sistematización

María Rocío Cifuentes-Patiño

\section{ARTÍCULOS}

Hacer lo que se sabe, pensar lo que se hace. La sistematización como modalidad investigativa Alfonso Torres-Carrillo

Aportes y desafios de la Sistematización de experiencias en el Trabajo Social y la extensión crítica. Apuntes y reflexiones desde la perspectiva de la Educación Popular

María Rosa Goldar

Valeria Chiavetta

La sistematización en Trabajo Social y la epistemología feminista del punto de vista. Diálogos sobre la producción de conocimiento sustentada en experiencias

Ruth Noemí Parola

María Florencia Linardelli

La Sistematización investigativa de las experiencias: del baile de los que sobran a la fiesta de los que faltan

María Belén Ortega-Senet

Sistematización y Trabajo Social en Chile. El largo y sinuoso camino

Patricia Lorena Castañeda-Meneses

Ana María Salamé-Coulon
Sentipensar la pandemia COVID-19 desde la sistematización de la experiencia en Trabajo Social: reflexiones del profesor Oscar Jara Holliday

Elia Sepúlveda-Hernández

La sistematización de experiencias, una investigación social cualitativa que potencia buenas prácticas de convivencia y gobierno. La experiencia de un conjunto residencial multifamiliar en Cali, Colombia Martha Lucia Echeverry-Velásquez Manuela Prada-Dávila

Construcción de subjetividades epistemológicaspolíticas de profesoras y profesores de Investigación social en una universidad privada y confesional en Bogotá

Giovanni Mora-Lemus

Sistematización de la experiencia Reconocimiento de los derechos humanos del adulto mayor en dos familias residentes en Cali y Valledupar (Colombia)

Lina María Cuello-Lacouture

Jimena del Pilar Jaramillo-Jaramillo

La memoria transformadora como estrategia de intervención profesional en los procesos de reconciliación social: comprensión a partir de mujeres campesinas, excombatientes y jóvenes en Manizales, Colombia

Yeimmy Stephania Corredor-Sotelo

Juliana Fuertes-Fuertes

Sistematización de una estrategia de educación informal implementada en personas privadas de la libertad en el establecimiento penitenciario de mediana seguridad y carcelario de Barranquilla, Colombia

Rafael Humberto Herrera-Mercado Rafael Alberto Zambrano-Vanegas 
Aportes significativos del proceso de intervención comunitaria con la Escuela Popular de Comunicación Alternativa Jaime Garzón de la ciudad de Cúcuta, Colombia

Carlos Lasso-Urbano

La sistematización de la intervención como metodología de investigación en Trabajo Social. Importancia práctica y teórica de la fase de recogida de datos en la intervención social según experiencia del Programa de Apoyo a las Familias en Zaragoza, España

Elisa Esteban-Carbonell

Nuria Del Olmo-Vicén

Papel de la sistematización de experiencias en los procesos de evaluación de intervenciones de salud pública en la Comuna Saludable por la Paz, Cali - Colombia

Jenny Faisury Peña-Varón

Paola Andrea Marín-Velásquez

Janeth Mosquera-Becerra

Experiencia de intervención social en hogares comunitarios integrales del barrio Alfonso Bonilla Aragón, Cali - Colombia

Julián Alexander Montaño-Cárdenas

Las políticas sociales y el gobierno de la "población indígena". Estrategias y regulaciones en el multiculturalismo chileno

Rodrigo Agustín Navarrete-Saavedra

Representaciones sociales sobre estilo de autoridad y tipos de interacción en cuidadores de residencias de protección infantil en Chile

Marcelo Gallegos-Fuentes
Carmen Gloria Jarpa-Arriagada

Reflexiones sobre inseguridad social y cuestiones penales. Una respuesta estratégica a partir de experiencias de cooperativismo con ex detenidos en Argentina

Analia Elizabeth Otero

Yael Yanina Barrera

Desarrollo y salud: la emergencia de un nuevo paradigma

Jesús María Sánchez-Ordóñez

Trabajo Social en ejercicio libre: la perspectiva profesional en España

Paula Frieiro-Padín

Tamara Fernández-Arias

Rubén González-Rodríguez

\section{RESEÑAS}

Social Work and the City: Urban Themes in 21stCentury Social Work

Felipe Saravia-Cortés

Respuestas del Trabajo Social ante emergencias sociales y problemáticas sociales complejas de México y España

Felipe Saravia-Cortés

El feminismo, el género y la profesionalización del trabajo social en Colombia (1936-2004)

Ambar Oriana Serna-Lombo

El puño invisible. Arte, revolución y un siglo de cambios culturales

Carlos Arturo Robledo-Marín

\section{PROSPECTIVA}

No. $31 \bullet$ ene.-jun. 2021

e-ISSN: 2389-993X • Universidad del Valle 